\section{Establishing a Pharmacy Practice for Today: Reflections from an Innovative Site \\ INTRODUCTION}

Pharmacy practice is no stranger to innovation: it has evolved from a dispensary-only practice to a clinical presence on virtually all patient care units, with a focus on patient-centred care. ${ }^{1}$ Pharmacy departments are forced to embrace innovation to keep up with today's expectations. The Alberta Health Services Pharmacy Services team at South Health Campus in Calgary, Alberta, was no exception to this expectation. When the South Health Campus was created, all teams were mandated to incorporate 4 foundational philosophies- patientand family-centred care, innovation, collaborative practice, and wellness - into their routine operational and clinical activities. This "out of the box" thinking resulted in an establishment designed with the expanding scope of pharmacy practice near the forefront, where every pharmacist practises to full scope, whether it is a patient's first acute care visit or a routine ambulatory clinic visit. Pharmacy services are available 24 hours per day, 7 days per week. In this correspondence, we review some of the key concepts considered in the creation of the innovative pharmacy practice at South Health Campus.

\section{WORKFORCE OPTIMIZATION}

One basic, yet crucial, concept embraced by the South Health Campus Pharmacy Services team is the importance of workforce optimization. Pharmacists with various backgrounds and levels of experience were recruited to create the team. Innovative community pharmacists were hired for their perspectives in primary care, and motivated hospital pharmacists were hired for their acute care experiences. New graduates were hired for their enthusiasm and fresh perspectives. The combination of practice backgrounds offered the team a wide range of therapeutic expertise and experience across the continuum of care, keeping the focus on patient- and family-centred care. Optimizing the pharmacy workforce also meant placing the right people in the right jobs at the right times. At South Health Campus, regulated technicians and pharmacy assistants practise to their full qualifications, covering dispensary and operational demands, which allows pharmacists to spend most of their time providing clinical services directly in patient care areas. Within the dispensary, a single pharmacist reviews orders for therapeutic appropriateness and triages clinical concerns.

\section{SETTING EXPECTATIONS}

Upon opening of the South Health Campus in 2012, another concept introduced to the Pharmacy Services team was the expectation that all staff would practice to full scope; as such, requirements for current and future hires were established in terms of practice skills. Authorization to order laboratory tests was required for all pharmacists upon employment. Additional prescribing authorization was not required upon commencement of employment, but all new hires pledged to obtain this designation. The same was initially true for injection certifications, although this skill is now mandated upon employment. Three years later, all 35 of the South Health Campus pharmacists have their injection certification, with an increasing number receiving additional prescribing authorization each year; the target is for $100 \%$ of pharmacists to have this authorization by December 31, 2016.

\section{PHARMACISTS AT EVERY STEP OF THE PATIENT'S JOURNEY}

The expectation that all staffing groups would be practising to full scope positioned the Pharmacy Services team to take a meaningful place in the integrated continuum of care. South Health Campus pharmacists are directly involved in patient care activities throughout the patient's journey, from their emergency department admission, during their acute care stay, and in follow-up within the ambulatory clinic. The pharmacists work in clinical teams, usually consisting of 3 or 4 pharmacists, dedicated to a therapeutic area. The teams rotate through inpatient and ambulatory care areas, which encourages development of diverse skills within their respective specialties, as well as enhancing their appreciation of the patient's journey. For example, the mental health team provides care for patients admitted to the mental health acute care units and ambulatory clinics. The decision to provide clinical and dispensary pharmacy services 24 hours per day was supported by the belief that pharmacists are valuable team members when they are present, and that their value to patients remains the same regardless of the time of day.

\section{ORGANIZATIONAL RESILIENCE}

With the knowledge that it would take more than individuals to sustain these innovative concepts, emphasis was placed on 
organizational resilience within the staffing structure. The Pharmacy Services team of South Health Campus invested substantial time into team-building and culture development activities before the facility opened. This fostered development of respect and established a culture of collaboration and accountability within the department. Time was also dedicated to integrating pharmacists within multidisciplinary teams, gaining support from other health care professionals, and ultimately providing better care for patients and their families.

\section{CONCLUSION}

The concepts of optimizing the workforce, expecting full scope and full coverage, being present at every step of the patient's journey, and developing organizational resilience have helped us to build a pharmacy practice within the South Health Campus institution that supports the sites foundational philosophies, bringing it one step closer to meeting today's ever-evolving expectations.

Reference

1. Bonnici A, Bornstein C, Bussières JF, Doucette D, Hall K, Jones R, et al., editors. Hospital pharmacy in Canada 2013/2014 report. Eli Lilly; 2015.

Josée Rioux, BScPharm, ACPR

Clinical Pharmacist

Pharmacy Services - Peter Lougheed Center

Alberta Health Services

Calgary, Alberta

Maria Anwar, BSCPharm, ACPR

Pharmacy Clinical Practice Leader

Pharmacy Services - South Health Campus

Alberta Health Services

Calgary, Alberta

Competing interests: None declared.

Acknowledgements: The authors would like to thank Rhonda Roedler and Susan Howlett-Wise for their contributions to this work. 